\title{
Intercalated Duct of the Pancreas
}

National Cancer Institute

\section{Source}

National Cancer Institute. Intercalated Duct of the Pancreas. NCI Thesaurus. Code C49267.

The tiny tubules with a very thin epithelial layer in the pancreas running from the pancreatic acini into the striated duct. 